\title{
Gradients of connectivity distance in the cerebral cortex of the macaque monkey
}

\author{
Sabine Oligschläger ${ }^{1,2,3} \cdot$ Ting Xu $^{4,5} \cdot$ Blazej M. Baczkowski $^{1,2,3,6} \cdot$ Marcel Falkiewicz $^{1} \cdot$ Arnaud Falchier $^{5} \cdot$ Gary Linn $^{5}$. \\ Daniel S. Margulies ${ }^{1,3,7}$
}

Received: 26 December 2017 / Accepted: 3 December 2018 / Published online: 13 December 2018

(c) The Author(s) 2018

\begin{abstract}
Cortical connectivity conforms to a series of organizing principles that are common across species. Spatial proximity, similar cortical type, and similar connectional profile all constitute factors for determining the connectivity between cortical regions. We previously demonstrated another principle of connectivity that is closely related to the spatial layout of the cerebral cortex. Using functional connectivity from resting-state fMRI in the human cortex, we found that the further a region is located from primary cortex, the more distant are its functional connections with the other areas of the cortex. However, it remains unknown whether this relationship between cortical layout and connectivity extends to other primate species. Here, we investigated this relationship using both resting-state functional connectivity as well as gold-standard tract-tracing connectivity in the macaque monkey cortex. For both measures of connectivity, we found a gradient of connectivity distance extending between primary and frontoparietal regions. In the human cortex, the further a region is located from primary areas, the stronger its connections to distant portions of the cortex, with connectivity distance highest in frontal and parietal regions. The similarity between the human and macaque findings provides evidence for a phylogenetically conserved relationship between the spatial layout of cortical areas and connectivity.
\end{abstract}

Keywords Cortical topography $\cdot$ Connectivity $\cdot$ Organizing principles $\cdot$ Primate phylogeny

\section{Introduction}

Cortical connectivity conforms to a series of organizing principles. Regions tend to be more interconnected when they lie close to each other (Betzel et al. 2016; Beul et al. 2015; Ercsey-Ravasz et al. 2013; Goulas et al. 2017; Kaiser

Electronic supplementary material The online version of this article (https://doi.org/10.1007/s00429-018-1811-1) contains supplementary material, which is available to authorized users.

Daniel S. Margulies

daniel.margulies@icm-institute.org

1 Max Planck Research Group for Neuroanatomy and Connectivity, Max Planck Institute for Human Cognitive and Brain Sciences, Leipzig, Germany

2 Faculty of Life Sciences, University of Leipzig, Leipzig, Germany

3 International Max Planck Research School NeuroCom, Leipzig, Germany

4 Center for the Developing Brain, Child Mind Institute, New York, NY 10022, USA and Hilgetag 2006; Roberts et al. 2016; Rubinov et al. 2015; Vértes et al. 2012), when they feature similar cortical microstructure (Barbas 2015; Beul et al. 2015, 2017; Goulas et al. 2016, 2017; Huntenburg et al. 2017; Pandya et al. 2015; Pandya and Sanides 1973; Pandya and Yeterian 1985), and when they share common connections to other cortical regions (Betzel et al. 2016; Costa et al. 2007; Song et al. 2014; Vértes et al. 2012). These principles are shared across a variety of species such as the mouse, cat, macaque, and

5 Center for Biomedical Imaging and Neuromodulation, Nathan Kline Institute for Psychiatric Research, Orangeburg, NY 10962, USA

6 Department of Neurology, Max Planck Institute for Human Cognitive and Brain Sciences, Leipzig, Germany

7 Centre National de la Recherche Scientifique (CNRS), UMR 7225, Frontlab, Institut du Cerveau et de la Moelle épinière, Hôpital Pitié-Salpêtrière, 47, boulevard de l'Hôpital, 75010 Paris, France 
human, demonstrating phylogenetically conserved patterns of connectivity in the mammalian brain.

In the human cerebral cortex, we previously described a principle of cortical organization that relates the patterns of connectivity to the spatial layout of cortical regions (Oligschläger et al. 2017). Using resting-state fMRI, we revealed a gradient of connectivity distance that progresses from primary sensory-motor areas to higher order association cortex. Specifically, the further a region is located from primary cortex, the more distant are its functional connections with the other areas of the cortex. Connectivity distance peaked in regions of the default mode network (DMN)—regions that underwent disproportionate expansion during primate phylogeny (Hill et al. 2010) and systematically occupy locations at maximal distance from primary cortex (Margulies et al. 2016).

Buckner and Krienen addressed the spatial distribution of connectivity distance in their 'tethering hypothesis' (2013) and attributed it to the cortical expansion that occurred during primate phylogeny. Expansion of the cortical sheet is presumed to have caused increasingly larger proportions of cortex to lie distant from developmental constraints that differentiate primary cortex (Buckner and Krienen 2013; Rosa and Tweedale 2005). While this link to expansion suggests a phylogenetically conserved relationship between cortical layout and connectivity in other primate species, a direct assessment in nonhuman primates is lacking. We hypothesize that connectivity distance follows a gradient that is anchored in the regions of primary cortex in the nonhuman primate, as well. It is, thus, our goal in the present study to examine the relationship between cortical layout and connectivity in a nonhuman primate species.

In light of our prior finding in the human cerebral cortex, here, we investigated whether the relationship between connectivity and cortical layout was consistent in both functional connectivity and gold-standard tract-tracing connectivity data in the macaque monkey. Functional connectivity was calculated based on resting-state fMRI data acquired in macaque monkeys (Milham et al. 2018). We obtained tracttracing data from a publicly available database of weighted and directed interareal connections based on a systematic large-scale anatomical investigation of the macaque cortex (Markov et al. 2013, 2014). For both types of connectivity, we assessed the geodesic distance along the cortical surface to highly connected areas. In keeping with the prior finding of a gradient extending from regions of primary cortex, we predicted that the further a cortical site is located from primary cortex, the longer its average connectivity distance. We found both the functional connectivity distance and structural connectivity distance to systematically vary as a function of distance from locations of primary cortex. In conjunction with our previous findings in the human cerebral cortex, the current study provides evidence for a phylogenetically conserved relationship between the spatial layout of cortical areas and connectivity.

\section{Materials and methods}

\section{Data}

\section{MRI data}

Anatomical and functional MRI data of the macaque monkey cortex were obtained from the publicly available NKI data set of the PRIMatE Data Exchange database (Milham et al. 2018). The Newcastle University cohort was included in the current study, consisting of 14 macaque monkeys (macaca mulatta) scanned on a primate-dedicated Vertical Bruker 4.7T scanner. ${ }^{1}$ Analysis was restricted to ten animals (eight males, age $=8.28 \pm 2.33$, weight $=11.76 \pm 3.38$ ), each of whom included two awake resting-state fMRI scans. The details of the data acquisition and experiment procedures have been described in the previous studies (Baumann et al. 2011, 2015; Poirier et al. 2017; Rinne et al. 2017; Schönwiesner et al. 2015; Slater et al. 2016; Wilson et al. 2015). In brief, two 8.33-min (250 volumes) sessions of resting-state fMRI data were acquired, while the animals were awake (resolution $=1.2 \mathrm{~mm}$ isotropic, $\mathrm{TE}=16 \mathrm{~ms}, \mathrm{TR}=2000 \mathrm{~ms}$ ), and structural T1-weighted images were acquired using MDEFT sequence $(0.6 \mathrm{~mm}$ isotropic resolution, $\mathrm{TE}=6 \mathrm{~ms}$, $\mathrm{TR}=750 \mathrm{~ms}$ ).

\section{Tract-tracing data}

Tract-tracing data of the macaque monkey cortex were obtained from the publicly available database Core-Nets (core-nets.org). Acquisition details have been described elsewhere (Markov et al. 2011, 2014). In brief, the macaque cortex was parcellated into 91 regions per hemisphere (M132 atlas). Across 28 macaque monkeys, retrograde tracers were injected into a subset of 29 regions. These tracers label neurons that project onto the injected region. Hence, we will, on occasion, refer to injections sites as projection sites, and regions of labeled neurons as source regions.

The current study used information on the number of labeled neurons per region for each injection. Geometric measures were taken from the cortical surface (at mid-thickness) representation of the Yerkes 19 group atlas in the form of a triangulated surface mesh including the M132 parcellation and injection sites (Donahue et al. 2016).

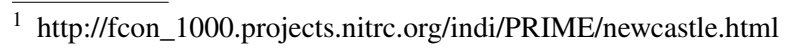




\section{Data processing}

The code for data processing and analysis has been made available online. $^{2}$

\section{MRI preprocessing}

Structural and functional image preprocessing have been described in detail in Xu et al. (2017). Structural processing comprised spatial noise removal, constructing a mean image from multiple T1-weighted images, brain extraction, tissue segmentation, reconstructing native surfaces, and surface registration from native space to the Yerkes19 group template surface (Donahue et al. 2016). The surfaces were downsampled to 10,242 vertices per surface.

Functional preprocessing was conducted using a customized version of Connectome Computation System for nonhuman primate (NHP) data (Xu et al. 2015). Temporal spikes were compressed and the time series were corrected for slice timing, motion, and field bias. Further steps included normalizing the 4D global mean intensity, regressing out nuisance signal (mean time series for WM and CSF masks, Friston-24 motion parameters), linear and quadratic trends, as well as band-passed $(0.01<f<0.1 \mathrm{~Hz})$ filtering. The functional volumes were registered to native anatomical space and projected onto the native middle surface, where they were spatially smoothed $(3 \mathrm{~mm}$ full width at half maximum) and downsampled to a 10k surface. The preprocessed scans were then concatenated for each monkey.

The functional data were quality controlled using framewise displacement (FD) (Power et al. 2012). We performed scan scrubbing by including time points with a mean FD below or equal to $0.2 \mathrm{~mm}$. The average mean FD across the included scans (10 animals $\times 2$ scans) was 0.066 $(\mathrm{SD}=0.018)$.

\section{Connectivity distance}

We computed connectivity distance both from tract-tracing and resting-state fMRI. While tract tracing reflects a goldstandard direct measure of cortico-cortical connections, the technique also remains limited for whole-brain studies by the number of injections. Connectivity measures obtained using resting-state fMRI, on the other hand, are inferred from correlated time courses and as such should be regarded as an indirect measure of connectivity. Its primary advantage is full-brain coverage. The conjunction of both measures allowed us to leverage the merits of each, complementing the sparse nature of tract tracing with full-brain coverage of resting-state fMRI, as well as validating the indirect measure

\footnotetext{
$\overline{2}$ https://github.com/soligschlager/distconnect_macaque/tree/master.
}

from resting-state fMRI against the gold-standard measure of connectivity from tract tracing.

\section{Functional connectivity distance}

Connectivity matrices were created separately for each hemisphere of each macaque monkey. Functional connectivity between each pair of cortical nodes was quantified by correlating their time series (Pearson product-moment correlation coefficient) and was entered in a node-by-node matrix of functional connectivity.

To capture the distance of a cortical site from its functionally connected areas within the cortical layout, we computed the average geodesic distance from each node of the native surface to its functionally connected nodes. The geodesic distance describes the shortest path along the cortical surface between two points allowing a direct assessment of the implications of relative spatial positions across the cortical surface. Geodesic distance was computed using an algorithm for approximating exact geodesic distance on triangular meshes (O'Rourke 1999) as implemented in the python surfdist and gdist packages.

The calculation of functional connectivity distance was identical to that described in our previous investigation into the human cortex (see Fig. 1 in Oligschläger et al. 2017). Connectivity was thresholded for each node by its $2 \%$ highest connectivity to determine the functionally connected nodes. Applying a node-wise threshold instead of defining an overall threshold attempts to adjust for differences in correlation strengths across nodes. For each node, the distance to $2 \%$ highest connected nodes was then averaged, yielding the overall functional connectivity distance for that node. Maps of functional connectivity distance were averaged over the monkeys and then averaged across nodes within each area of the parcellation.

\section{Structural connectivity distance}

To capture the distance of a cortical site from its source neurons, we calculated the geodesic distance from the injection sites to their labeled neurons. As the precise location of each labeled neuron was not reported, we instead used the centroid of the corresponding area as spatial reference for the labeled neurons. We only included labeled neurons extrinsic to respective injected area. For each injection site, we then took the average geodesic distance from all its source neurons as a summary measure of its connectivity distance (Fig. 1). As source neurons within the same area shared the same spatial reference location, this is equivalent to an area's average geodesic distance from its source areas weighted by the respective number of source neurons. 


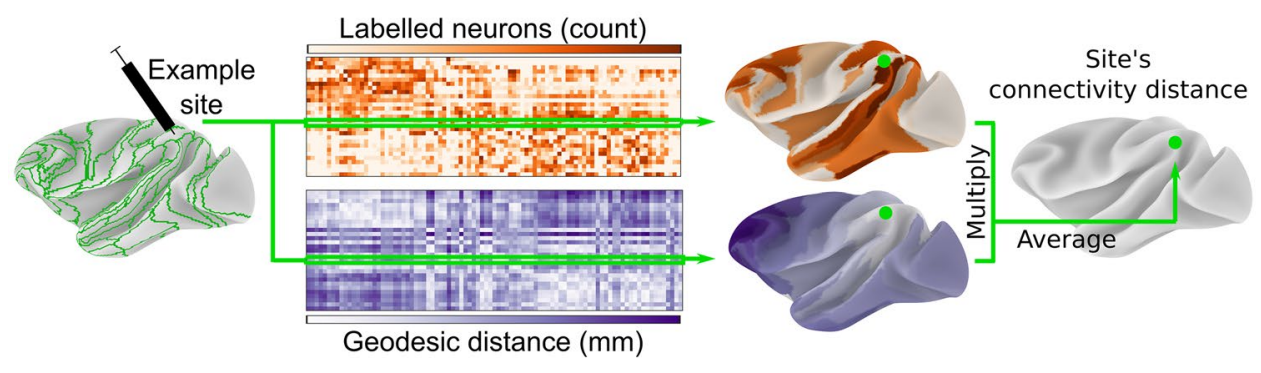

Fig. 1 Structural connectivity distance captures the average distance of a cortical site from its source neurons. Retrograde tract-tracing data for 29 injected regions of the M132 macaque cortical atlas (green outlines) were obtained from Core-Nets (Markov et al. 2011, 2014). Geodesic distance was calculated along the cortical surface of

\section{Data analysis}

We next investigated the topography of connectivity distance. Specifically, we asked whether a cortical site's connectivity distance is related to that site's distance from primary sensory-motor areas. Based on our hypothesis that the spatial distribution of connectivity distance follows a gradient anchored in primary cortex, we predicted that the further a cortical site is located from primary cortex, the longer its connectivity distance. For both the functional and structural connectivity distance, we tested these predictions using a general linear model (GLM), with an area's geodesic distance from the border of the closest primary area as independent variable, and its connectivity distance as the dependent variable.

As locations of primary cortex (Fig. 2), we included primary visual (V1), primary auditory (Core), primary somatosensory (area 3), and primary motor areas (F1). We excluded olfactory and gustatory primary cortex from our analyses, because they constitute chemosensory systems with distinct patterns of laminar differentiation. Unlike primary areas of visual, auditory, and somatosensory cortex, those of olfactory and gustatory cortex do not exhibit the highly differentiated structure of koniocortex (Johnson et al. 2000; Pritchard 2012; van Hartevelt and Kringelbach 2012). While the primary areas of visual, auditory, and somatosensory systems give rise to processing hierarchies that sequentially convey information about the external environment to the least differentiated limbic structures, the chemosensory systems reflect a closer relationship to the limbic structures (Mesulam 1998), and thus depart from the relationship hypothesized by the tethering hypothesis.

For functional connectivity distance, the GLM expressed the spatial distribution of the average connectivity distance per area as a function of an area's average geodesic distance from the closest border of primary sensory-motor areas. A major advantage of the functional connectivity here is the the Yerkes19 group atlas (Donahue et al. 2016). For each injection, the connectivity distance was calculated by the injection site's average geodesic distance from all area centroids weighted by the count of labeled neurons per area. Area centroids were used, because the precise location of each labeled neuron was unknown

whole-brain coverage (compared to 29 cortical samples in the tract-tracing data).

Similarly, the spatial distribution of structural connectivity distance was modeled as a function of distance from primary cortex. Here, the geodesic distance of each injection site to the closest border of primary sensory-motor areas served as the independent variable in the GLM. Two covariates of no interest were included. First, as we only included labeled neurons extrinsic to the injected area, injections into larger areas will have increased connectivity distance solely by virtue of the methodological setup. We thus corrected for region size as a confounding factor by including the number of nodes in the injected region as a covariate in the GLM. Second, some areas are positioned within the cortical geometry to have overall longer distances to the rest of the cortex than geometrically more central areas. The effect of position within the overall geometry of the cortex was thus measured as the average distance of an injection site from all cortical nodes and added as a covariate to the GLM.

In addition to the hypothesis-driven analysis describing the spatial distribution of connectivity distance with

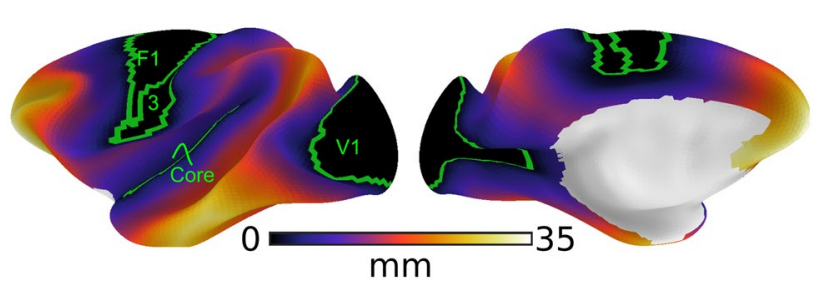

Fig. 2 Distance from primary sensory-motor cortex. The map shows the geodesic distance from the closest border of a primary cortical region [green outline: primary visual (V1), primary auditory (Core), primary somatosensory (area 3), and primary motor areas (F1)]. Geodesic distance describes the shortest path along the cortical surface and was used to assess of the implications of relative spatial positions across the cortical surface. The distance from primary cortex served as the predictor variable in GLMs assessing whether the spatial distribution of connectivity distance follows a gradient anchored in primary cortex 
A

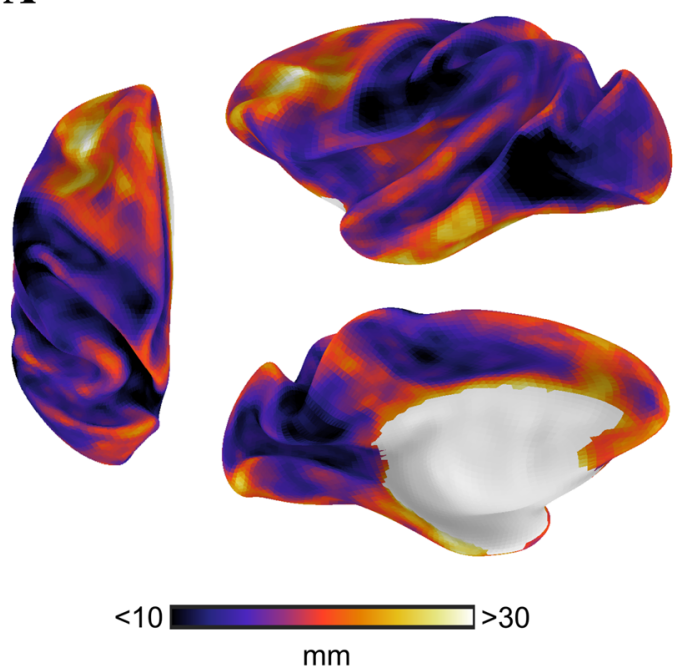

Fig. 3 Spatial distribution of functional connectivity distance (a) was investigated with relation to locations of primary cortex. b Each area's mean functional connectivity distance is plotted against its mean distance from primary cortex. Functional connectivity distance systematically varied across the cortex as a function of distance from primary cortex demonstrated by the significant relationship between

respect to locations of primary cortex, we conducted a search procedure to check for the possibility that the distance from a different set of regions might account better for the spatial distribution of structural connectivity distance. To this end, we randomly sampled cortical locations 100,000 times and repeated the above-described GLM using these locations as anchor points instead. To identify good reference locations, we calculated each node's mean variance explained across all GLMs that included this node in their random set of anchors. The resulting map of average variance explained provides a hypothesis-free estimate of locations that anchor the spatial distribution of connectivity distance.

However, probing the search space of all possible sets of reference nodes by random sampling poses a huge combinatorial problem which we addressed and replaced in a second and separate analysis that included optimization. Starting with a random set of nodes (size between 3 and 5), these nodes were moved in steps of $1 \mathrm{~mm}$ towards the direction of higher variance explained until a stable set of locations was found (i.e., where further moving by $1 \mathrm{~mm}$ did not result in higher variance explained). This search was repeated 1000 times, each time starting with a new random set of nodes. For each node, we counted the number of times which it was included in the final and stable sets of reference nodes. The resulting map of counts distinguishes between positive (the further from reference location, the longer the connectivity distance) and negative (the further from reference location, the shorter the connectivity distance) relationships.
B

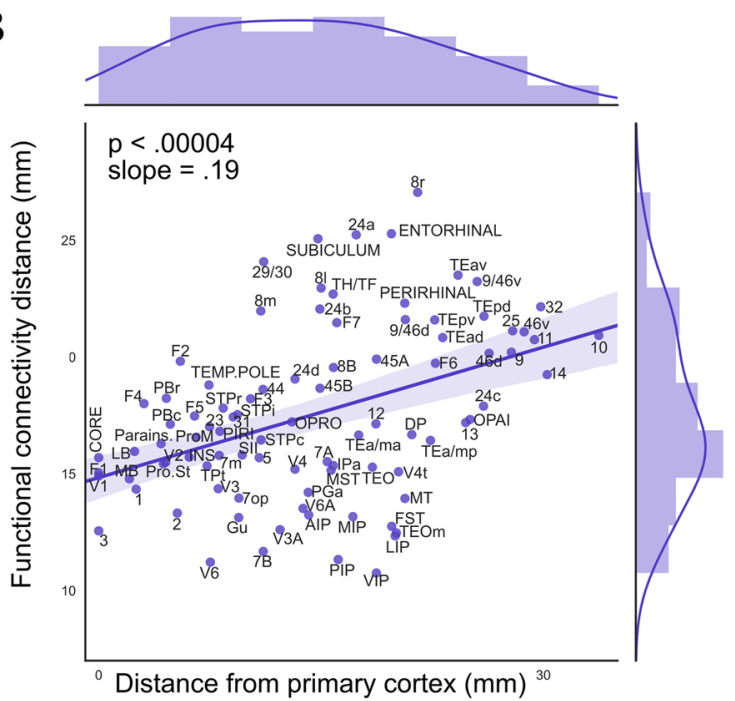

the map of distance from primary cortex (shown in Fig. 2) and connectivity distance (a) $\left(F_{1.89}=18.8, p<0.00004, R^{2}=0.17 ; t_{90}=4.3\right.$, $p<0.00004, \beta=0.19,95 \% \mathrm{CI}=[0.10,0.27])$. These findings show that the further a node was located from primary cortex, the longer its functional connectivity distance

\section{Results}

\section{Functional connectivity distance follows a gradient anchored in primary cortex}

We investigated the spatial distribution of connectivity distance based on resting-state functional connectivity in relation to locations of primary cortex. Connectivity distance was the shortest in sensory-motor regions and peaked in higher-order association regions in lateral and medial frontoparietal cortex (Fig. 3a). Information on each area's connectivity distance can be found in Online Resource Fig. 1.

We found functional connectivity distance to systematically vary as a function of distance from primary cortex. A GLM with distance from primary cortex as independent variable and connectivity distance as dependent variable $\left(F_{1,89}\right.$ $\left.=18.8, p<0.00004, R^{2}=0.17\right)$ showed that the distance from primary regions significantly accounted for the spatial distribution of functional connectivity distance $\left(t_{90}=4.3\right.$, $p<0.00004, \beta=0.19,95 \%$ CI [0.10, 0.27]). Specifically, the further a node was located from primary cortex, the longer its functional connectivity distance (Fig. 3b).

By an arbitrary threshold of studentized residuals larger than 2, the functional connectivity distance of areas VIP, 8r, as well as cortical limbic regions 24a, 29/30, subiculum, and entorhinal cortex were found to deviate from the overall pattern. While VIP is at medium distance from primary cortex, it had among the shortest functional connectivity distance. Other intraparietal areas (PIP, LIP, and MIP) shared this 
A

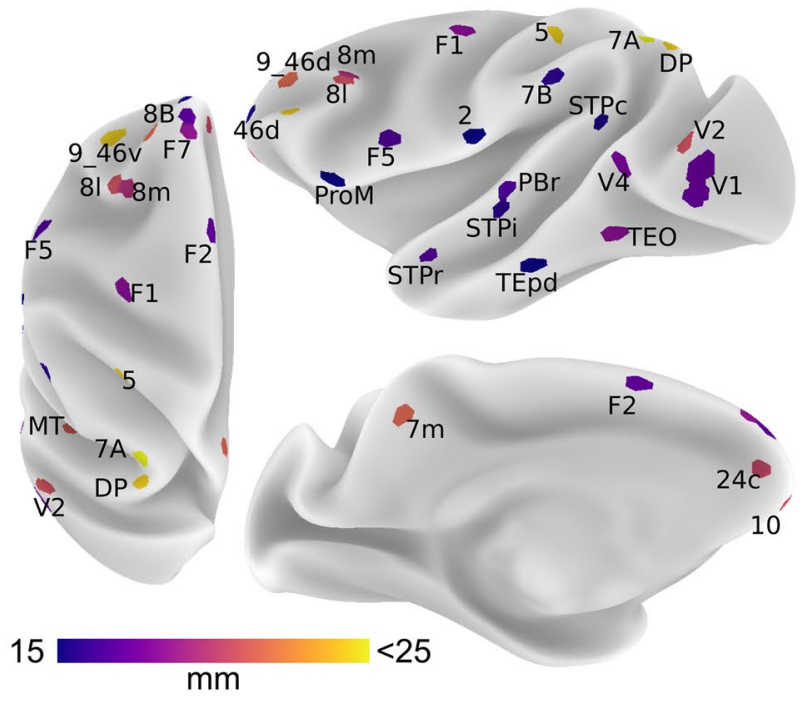

Fig. 4 Spatial distribution of structural connectivity distance (a) was investigated with relation to locations of primary cortex. Structural connectivity distance was corrected for confounding factors of region size and location. b Structural connectivity distance systematically varied across the cortex as a function of distance from primary

property but deviated less. In contrast, area $8 \mathrm{r}$ and the cortical limbic regions had much longer functional connectivity distance than predicted by their distance from primary cortex. Information on each area's deviation from the overall relationship is presented in Online Resource Fig. 2.

\section{Structural connectivity distance follows a gradient anchored in primary cortex}

The spatial distribution of structural connectivity distance was also inversely related to distance from primary cortex. While the functional connectivity analysis was calculated across the entire cortex, the number of injection sites restricted our analysis to the spatial distribution of injection sites with respect to primary cortical areas.

Structural connectivity distance was the shortest in unimodal cortex and the highest in frontoparietal regions of higher order association cortex (Fig. 4a), and followed a gradient extending from primary cortical areas. The GLM $\left(F_{3,25}=10.01, p<0.0002, R^{2}=0.55\right)$ revealed a significant relationship between structural connectivity distance and distance from primary cortex. Connectivity distance systematically increased with distance from primary cortex $\left(t_{26}=2.12, p<0.05, \beta=0.18,95 \%\right.$ CI $\left.[0.005,0.35]\right)$. Thus, the further an injection site was located from primary cortex, the longer its structural connectivity distance (Fig. 4b). Information on each injection site's connectivity distance can be found in Online Resource Fig. 1.
B

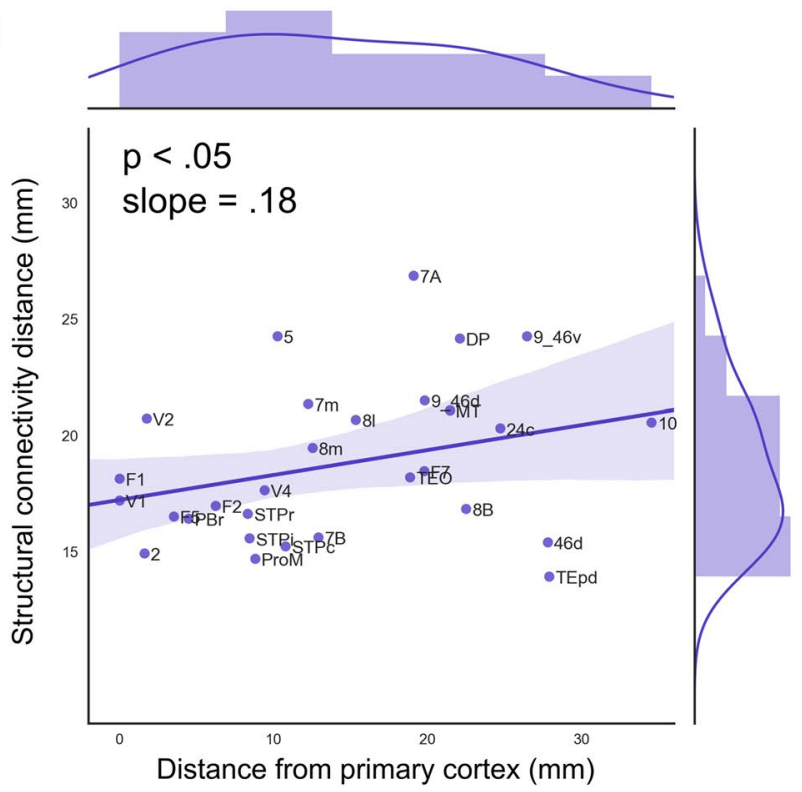

cortex demonstrated by the significant relationship between the map of distance from primary cortex (shown in Fig. 2) and connectivity distance (a) $\left(F_{3,25}=10.01, p<0.0002, R^{2}=0.55 ; t_{26}=2.12, p<0.05\right.$, $\beta=0.18,95 \%$ CI $[0.005,0.35])$

The structural connectivity distance of areas TEpd and 7A deviated from the overall pattern (by a studentized residual $>2$ ). While TEpd is far from primary cortex, it had among the shortest structural connectivity distances. In contrast, area 7A had the longest structural connectivity distance despite not being the furthest away from primary cortex. Both areas also differed substantially in their structural and functional connectivity distance. In the functional analysis, these areas follow the trend with respect to location of primary cortex more closely. Further information on deviations from the overall relationship is presented in Online Resource Fig. 2.

\section{Spatial distribution of structural connectivity distance (search procedure)}

In addition to the hypothesis-driven analysis describing the spatial distribution of connectivity distance with respect to locations of primary cortex, we conducted a search procedure to check for the possibility that a different set of regions might account better for the spatial distribution of the structural connectivity distance. A map of the average variance explained across nodes was obtained from 100,000 GLMs. Instead of distance from primary cortex, these GLMs included the distance from random locations as independent variable. The map (Fig. 5) identifies regions for which the distance from them either positively (red) or negatively (blue) accounts for the distribution of structural connectivity distance. These maps offer 


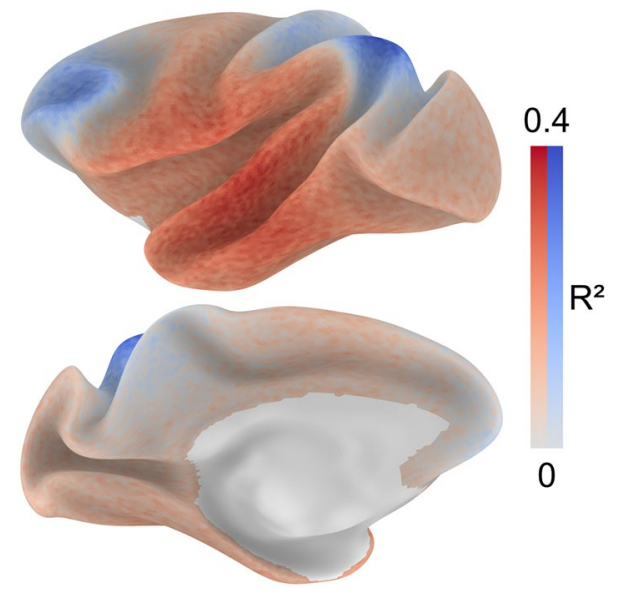

Fig. 5 Hypothesis-free search for anchor regions. To estimate locations that anchor the distribution of structural connectivity distance, 100,000 GLMs were repeated using distance from random sets of locations as the predictor variable. For each surface node, we calculated the average variance explained across the GLMs that included distance from a given node as the predictor. The map identifies regions from which the distance either positively (red) or negatively (blue) accounts for the distribution of structural connectivity distance. These maps offer an estimate of the whole-brain topography of structural connectivity distance based on the subset of known injection sites

an estimate of the whole-brain topography of structural connectivity distance based on the subset of known injection sites.

A comparison of functional connectivity distance with structural connectivity distance (Online Resource Fig. 3) and with estimated whole-brain structural connectivity distance (Online Resource Fig. 4) did not reveal a similarity across modalities. While the topography is partly consistent with that of the available whole-brain map of functional connectivity distance, differences pertain to regions of occipitoparietal and temporal cortex. Specifically, areas V2, MT, DP, 7A, 5, $7 \mathrm{~m}$, and area TEpd deviated the most from an ideal linear fit between functional and structural connectivity distances (Online Resource Fig. 3).

A separate search optimization procedure confirmed these findings. 1000 random locations were moved in 1-mm steps along the cortical sheet until a stable set of locations was found that best predicted the distribution of connectivity distance. We counted the number of times each node was included in the stable set of reference nodes (Online Resource Fig. 5). Peak regions confirm with the reported regions from the initial search procedure.

\section{Discussion}

The current study demonstrates a relationship between the spatial layout and connectivity of the macaque monkey cortex. The conjunction of tract-tracing and resting-state functional connectivity data allowed us to leverage the merits of each, complementing the sparse nature of tract tracing with full-brain coverage of resting-state fMRI, as well as validating the indirect measure from resting-state fMRI against the gold-standard measure of connectivity from tract-tracing. Characterizing a region's connectivity distance-measured as a region's average distance to connected areas-we found that the spatial distribution of connectivity distance follows a gradient that is anchored in primary cortex. While a direct similarity between structural and functional connectivity distance is only partly present, both measures follow this gradient. Specifically, the further a region is located from primary cortex, the stronger its connections to distant portions of the cortex. This topographic pattern is consistent with our previous findings in the human cortex (Oligschläger et al. 2017) offering an evolutionary perspective on brain organization.

The topography of connectivity distance in the macaque monkey cortex presented here coincides with that of the human cortex (Oligschläger et al. 2017). While deviations were observed in the lateral temporal and cingulate cortex across species, the overall similar topography of connectivity distance between the macaque and human cortex suggests an organizational principle of cortical connectivity. This correspondence opens up the possibility of a phylogenetically conserved relationship between connectivity and the spatial layout in the cortex. Nevertheless, given the substantial phylogenetic distance between humans and macaque monkeys, future studies are needed to investigate this relationship in across a broader spectrum of primate as well as other mammalian species.

An evolutionary perspective on cortical organization has been proposed by Buckner and Krienen (2013) who attribute the distribution of connectivity distance to the cortical expansion that occurred during primate phylogeny. Indeed, the topography of connectivity distance in both the macaque and human cortex closely concurs with the patterns of phylogenetic expansion (Chaplin et al. 2013; Fjell et al. 2015; Hill et al. 2010). According to the 'tethering hypothesis' (Buckner and Krienen 2013), cortical expansion during primate evolution has caused increasingly larger amounts of cortex to lie distant from primary cortex. Untethered from developmental constraints that differentiate primary cortex, these regions form unique characteristics of association cortex, such as their long-range connections.

Alongside the overall agreement between connectivity distance in the macaque and human cortex, we found a notable difference in the lateral temporal cortex of the macaque. While this region was among those with the longest functional connectivity distance in both the human and macaque monkey, it had comparatively short structural connectivity distance in the macaque. Although this difference between species may be confounded by differences in methodologies, 
this observation would be consistent with previously reported differences between human and macaque neuroanatomy. The human temporal lobe is disproportionately larger (Rilling and Seligman 2002) and marked by strongly modified projection patterns of the arcuate fasciculus (Rilling et al. 2008).

Further differences between the topography of macaque and human connectivity distance include posterior cingulate cortex, rostral anterior cingulate cortex (ACC), and posterior insula. In the human cortex, these regions deviate from the overall pattern and show relatively short connectivity distance compared to surrounding regions (Oligschläger et al. 2017). These deviations were less evident in the macaque. However, in the macaque too, the insula is marked by relatively short connectivity distance similar to sensory-motor regions, and the ACC exhibits a faint decrease in connectivity distance.

Our findings join a body of research into organizing principles that govern cortical connectivity of the mammalian brain. The distance between cortical sites is a major determinant of cortical connectivity, with connectivity strength between regions systematically decreasing with the distance between them (Betzel et al. 2016; Ercsey-Ravasz et al. 2013; Roberts et al. 2016). The findings presented here suggest that connectivity distance is modulated by a region's offset from primary cortex. Specifically, the rate by which connectivity strength decreases with the distance between regions might be modulated by how far they are located from primary cortex. As the connectivity strength has been shown to follow an exponential decay with distance (Ercsey-Ravasz et al. 2013), we expect this decay to be steepest in primary sensory-motor areas, becoming progressively less steep with distance from these areas.

The current findings provide spatial context in which organizing principles operate and might prove useful for integrating these principles with potential developmental mechanisms that give rise to them in the first place. A promising line of research models spatiotemporal dynamics during cortical ontogeny to reproduce major characteristics of connectivity. For example, assuming axons connect with the first neuron which they encounter, models of random axonal outgrowth successfully reproduced patterns of decreasing connectivity with distance (Kaiser et al. 2009). In a serial version of this model, neurons that started axon growth early formed longer connections than later ones (Lim and Kaiser 2015). This is of special relevance for the current findings given that neurons destined for association areas migrate earlier during cortical ontogeny than primary cortical areas (Fig. 2 in Rakic 2002).

Postnatal cortical maturation follows an opposite spatiotemporal pattern. While sensory-motor regions mature early during childhood, it is association cortex that fully matures last (Casey et al. 2005; Chomiak and Hu 2017; Toga et al. 2006). This spatiotemporal trajectory characterizes the maturation of structural properties such as gray matter density (Gogtay et al. 2004), thickness (Sowell et al. 2004), and surface expansion (Hill et al. 2010), as well as synaptic density (Huttenlocher 1990) and myelination (Flechsig 1920). When association cortex reaches maturity, it is marked by a more elaborate and complex microstructure than sensorymotor cortex. For example, pyramidal neurons in association cortex are larger (Elston et al. 1999) and possess both a larger number as well as longer dendrites (Elston et al. 2001). It has been proposed that these structural properties allow association regions to integrate larger numbers of inputs compared to primary sensory-motor regions (Elston 2002). The current findings provide further support for this proposal by indicating that association cortex integrates input from more widespread cortical regions.

Thalamocortical connectivity, established during cortical ontogeny, is another candidate mechanism that might define the observed topography of connectivity distance. While unimodal sensory-motor regions receive input from their specific thalamic nuclei, distributed regions of association cortex share thalamic input from the medial pulvinar nucleus (Goldman-Rakic 1988). For unimodal regions, it has been shown that thalamic input determines activity-dependent differentiation of sensory hierarchies (Chou et al. 2013; Katz and Shatz 1996; Lokmane et al. 2013). An interesting possibility is to consider a similar mechanism for the distributed regions of association cortex. These, too, might interconnect in a similar activity-dependent manner via their common thalamic input (Buckner and Krienen 2013; Shipp 2003). In conjunction with such models, an understanding of basic organizational principles of cortical connectivity can inspire future investigations that study these principles as an emergent property of spatiotemporal processes during cortical ontogeny.

The emergence of areas over the course of cortical phylogenesis might further account for the observed topography of connectivity distance. A connectivity 'blueprint' may be established during cortical evolution, such that regions predominantly connect with those already present when they emerge. Hence, a general tendency might be that connections are established mainly to regions that are phylogenetically older, but not vice versa. In that sense, the phylogenetically more recent regions of association cortex would be expected to connect both to sensory-motor cortex and among them, while sensory-motor areas connect primarily within their cortical hierarchies. Such scenario would imply a dissociation between sensory-motor and association cortex with respect to afferent and efferent connectivity distance. While association cortex would be marked by both long efferents and afferents, sensory-motor cortex would be marked by long afferents and short efferents. As the sequence of emerging areas during cortical evolution is a controversial question 
(cf. Sanides 1969), future studies that incorporate measures of the directionality of connections could help shed further light on such questions.

From a functional perspective, connectivity distance might index a region's hierarchical level and integrative capacity of information processing. For example, prior findings from Wagstyl and colleagues (2015) demonstrate that geodesic distance from primary cortical areas traces structural hierarchies in the macaque monkey cortex. Assuming that the level of global connectedness of a region contributes to its capacity of information integration, connectivity distance may be a structural feature that affords the hierarchies of information integration in the cortex.

To conclude, we reported on a phylogenetically conserved relationship between cortical layout and connectivity of the primate cortex. In the human cortex (Oligschläger et al. 2017), we revealed a gradient of connectivity distance extending between primary and frontoparietal regions. The further a region is located from primary sensory-motor cortex, the stronger its connections to distant portions of the cortex. Together, these findings highlight the utility of crossspecies comparative studies for addressing the phylogeny of cortical organization.

Acknowledgements Open access funding provided by Max Planck Society.

\section{Compliance with ethical standards}

Conflict of interest The authors declare that they have no conflicts of interest.

Ethical approval The authors have no conflicts of interest to declare. All data used in the current study are openly available and were previously acquired in accordance with the ethical standards of respective institutions.

Open Access This article is distributed under the terms of the Creative Commons Attribution 4.0 International License (http://creativeco mmons.org/licenses/by/4.0/), which permits unrestricted use, distribution, and reproduction in any medium, provided you give appropriate credit to the original author(s) and the source, provide a link to the Creative Commons license, and indicate if changes were made.

\section{References}

Barbas H (2015) General cortical and special prefrontal connections: principles from structure to function. Annu Rev Neurosci 38:269-289

Baumann S, Griffiths TD, Sun L, Petkov CI, Thiele A, Rees A (2011) Orthogonal representation of sound dimensions in the primate midbrain. Nat Neurosci 14(4):423-425

Baumann S, Joly O, Rees A, Petkov CI, Sun L, Thiele A, Griffiths TD (2015) The topography of frequency and time representation in primate auditory cortices. eLife 4:e03256
Betzel RF, Avena-Koenigsberger A, Goñi J, He Y, de Reus MA, Griffa A, Sporns O (2016) Generative models of the human connectome. NeuroImage 124(Pt A):1054-1064

Beul SF, Grant S, Hilgetag CC (2015) A predictive model of the cat cortical connectome based on cytoarchitecture and distance. Brain Struct Funct 220(6):3167-3184

Beul SF, Barbas H, Hilgetag CC (2017) A Predictive Structural Model of the Primate Connectome. Sci Rep 7:43176

Buckner RL, Krienen FM (2013) The evolution of distributed association networks in the human brain. Trends Cogn Sci 17(12):648-665

Casey BJ, Tottenham N, Liston C, Durston S (2005) Imaging the developing brain: what have we learned about cognitive development? Trends Cogn Sci 9(3):104-110

Chaplin TA, Yu H-H, Soares JGM, Gattass R, Rosa MGP (2013) A conserved pattern of differential expansion of cortical areas in simian primates. J Neurosci 33(38):15120-15125

Chomiak T, Hu B (2017) Mechanisms of hierarchical cortical maturation. Front Cell Neurosci 11:272

Chou S-J, Babot Z, Leingärtner A, Studer M, Nakagawa Y, O'Leary DDM (2013) Geniculocortical input drives genetic distinctions between primary and higher-order visual areas. Science 340(6137):1239-1242

Costa LdaF, Kaiser M, Hilgetag CC (2007) Predicting the connectivity of primate cortical networks from topological and spatial node properties. BMC Syst Biol. https://doi. org/10.1186/1752-0509-1-16

Donahue CJ, Sotiropoulos SN, Jbabdi S, Hernandez-Fernandez M, Behrens TE, Dyrby TB, Coalson T, Kennedy H, Knoblauch K, Van Essen DC, Glasser MF (2016) Using diffusion tractography to predict cortical connection strength and distance: a quantitative comparison with tracers in the monkey. J Neurosci 36(25):6758-6770

Elston GN (2002) Cortical heterogeneity: implications for visual processing and polysensory integration. J Neurocytol 31(3-5):317-335

Elston GN, Tweedale R, Rosa MG (1999) Cortical integration in the visual system of the macaque monkey: large-scale morphological differences in the pyramidal neurons in the occipital, parietal and temporal lobes. Proc Biolog Sci R Soc 266(1426):1367-1374

Elston GN, Benavides-Piccione R, DeFelipe J (2001) The pyramidal cell in cognition: a comparative study in human and monkey. $\mathbf{J}$ Neurosci 21(17):RC163

Ercsey-Ravasz M, Markov NT, Lamy C, Van Essen DC, Knoblauch K, Toroczkai Z, Kennedy H (2013) A predictive network model of cerebral cortical connectivity based on a distance rule. Neuron 80(1):184-197

Fjell AM, Westlye LT, Amlien I, Tamnes CK, Grydeland H, Engvig A, Walhovd KB (2015) High-expanding cortical regions in human development and evolution are related to higher intellectual abilities. Cereb Cortex 25(1):26-34

Flechsig PE (1920) Anatomie des menschlichen Gehirns und Rückenmarks auf myelogenetischer Grundlage (Vol. 1). G. Thieme

Gogtay N, Giedd JN, Lusk L, Hayashi KM, Greenstein D, Vaituzis AC, Thompson PM (2004) Dynamic mapping of human cortical development during childhood through early adulthood. Proc Natl Acad Sci USA 101(21):8174-8179

Goldman-Rakic PS (1988) Topography of cognition: parallel distributed networks in primate association cortex. Annu Rev Neurosci 11:137-156

Goulas A, Werner R, Beul SF, Saering D, van den Heuvel M, Triarhou LC, Hilgetag CC (2016) Cytoarchitectonic similarity is a wiring principle of the human connectome. bioRxiv. https://doi. org/10.1101/068254 
Goulas A, Uylings HBM, Hilgetag CC (2017) Principles of ipsilateral and contralateral cortico-cortical connectivity in the mouse. Brain Struct Funct 222(3):1281-1295

Hill J, Inder T, Neil J, Dierker D, Harwell J, Van Essen D (2010) Similar patterns of cortical expansion during human development and evolution. Proc Natl Acad Sci USA 107(29):13135-13140

Huntenburg JM, Bazin P-L, Goulas A, Tardif CL, Villringer A, Margulies DS (2017) A systematic relationship between functional connectivity and intracortical myelin in the human cerebral cortex. Cereb Cortex 27(2):981-997

Huttenlocher PR (1990) Morphometric study of human cerebral cortex development. Neuropsychologia 28(6):517-527

Johnson DMG, Illig KR, Behan M, Haberly LB (2000) New features of connectivity in piriform cortex visualized by intracellular injection of pyramidal cells suggest that "primary" olfactory cortex functions like "association" cortex in other sensory systems. J Neurosci 20(18):6974-6982

Kaiser M, Hilgetag CC (2006) Nonoptimal component placement, but short processing paths, due to long-distance projections in neural systems. PLoS Comput Biol 2(7):e95

Kaiser M, Hilgetag CC, van Ooyen A (2009) A simple rule for axon outgrowth and synaptic competition generates realistic connection lengths and filling fractions. Cereb Cortex 19(12):3001-3010

Katz LC, Shatz CJ (1996) Synaptic activity and the construction of cortical circuits. Science 274(5290):1133-1138

Lim S, Kaiser M (2015) Developmental time windows for axon growth influence neuronal network topology. Biol Cybern 109(2):275-286

Lokmane L, Proville R, Narboux-Nême N, Györy I, Keita M, Mailhes C, Garel S (2013) Sensory map transfer to the neocortex relies on pretarget ordering of thalamic axons. Curr Biol 23(9):810-816

Margulies DS, Ghosh SS, Goulas A, Falkiewicz M, Huntenburg JM, Langs G, Smallwood J (2016) Situating the default-mode network along a principal gradient of macroscale cortical organization. Proc Natl Acad Sci USA 113(44):12574-12579

Markov NT, Misery P, Falchier A, Lamy C, Vezoli J, Quilodran R, Knoblauch K (2011) Weight consistency specifies regularities of macaque cortical networks. Cereb Cortex 21(6):1254-1272

Markov NT, Ercsey-Ravasz M, Lamy C, Ribeiro Gomes AR, Magrou L, Misery P, Kennedy H (2013) The role of long-range connections on the specificity of the macaque interareal cortical network. Proc Natl Acad Sci 110(13):5187-5192

Markov NT, Ercsey-Ravasz MM, Gomes R, Lamy AR, Magrou C, Vezoli J, Kennedy H (2014) A weighted and directed interareal connectivity matrix for macaque cerebral cortex. Cereb Cortex 24(1):17-36

Mesulam MM (1998) From sensation to cognition. Brain A J Neurol 121(Pt 6):1013-1052

Milham MP, Ai L, Koo B, Xu T, Amiez C, Balezeau F, Baxter MG, Blezer ELA, Brochier T, Chen A, Croxson PL, Damatac CG, Dehaene S, Everling S, Fair DA, Fleysher L, Freiwald W, Froudist-Walsh S, Griffiths TD, Guedj C, Hadj-Bouziane F, Ben Hamed S, Harel N, Hiba B, Jarraya B, Jung B, Kastner S, Klink PC, Kwok SC, Laland KN, Leopold DA, Lindenfors P, Mars RB, Menon RS, Messinger A, Meunier M, Mok K, Morrison JH, Nacef J, Nagy J, Rios MO, Petkov CI, Pinsk M, Poirier C, Procyk E, Rajimehr R, Reader SM, Roelfsema PR, Rudko DA, Rushworth MFS, Russ BE, Sallet J, Schmid MC, Schwiedrzik CM, Seidlitz J, Sein J, Shmuel A, Sullivan EL, Ungerleider L, Thiele A, Todorov OS, Tsao D, Wang Z, Wilson CRE, Yacoub E, Ye FQ, Zarco W, Zhou Y-di, Margulies DS, Schroeder CE (2018) An open resource for non-human primate imaging. Neuron 100(1):61-74

O'Rourke J (1999) Computational Geometry Column 35. SIGACT News 30(2):31-32
Oligschläger S, Huntenburg JM, Golchert J, Lauckner ME, Bonnen T, Margulies DS (2017) Gradients of connectivity distance are anchored in primary cortex. Brain Struct Funct 222(5):2173-2182

Pandya DN, Sanides F (1973) Architectonic parcellation of the temporal operculum in rhesus monkey and its projection pattern. Z Anat Entwickl Gesch 139:127-161

Pandya DN, Yeterian EH (1985) Architecture and Connections of Cortical Association Areas. In: Peters A, Jones EG (eds) Association and Auditory Cortices. Springer, US, Boston, MA, pp 3-61

Pandya DN, Seltzer B, Petrides M, Cipolloni PB (2015) Cerebral cortex: architecture, connections, and the dual origin concept. Oxford University Press, Oxford

Poirier C, Baumann S, Dheerendra P, Joly O, Hunter D, Balezeau F, Griffiths TD (2017) Auditory motion-specific mechanisms in the primate brain. PLoS Biol 15(5):e2001379

Power JD, Barnes KA, Snyder AZ, Schlaggar BL, Petersen SE (2012) Spurious but systematic correlations in functional connectivity MRI networks arise from subject motion. NeuroImage 59(3):2142-2154

Pritchard TC (2012) Chapter 33-gustatory system. In: Mai JK, Paxinos G (eds) The human nervous system (Third Edition). Academic Press, San Diego, pp 1187-1218

Rakic P (2002) Neurogenesis in adult primate neocortex: an evaluation of the evidence. Nat Rev Neurosci 3(1):65-71

Rilling JK, Seligman Ra (2002) A quantitative morphometric comparative analysis of the primate temporal lobe. J Hum Evol 42(5):505-533

Rilling JK, Glasser MF, Preuss TM, Ma X, Zhao T, Hu X, Behrens TEJ (2008) The evolution of the arcuate fasciculus revealed with comparative DTI. Nat Neurosci 11(4):426-428

Rinne T, Muers RS, Salo E, Slater H, Petkov CI (2017) Functional imaging of audio-visual selective attention in monkeys and humans: how do lapses in monkey performance affect cross-species correspondences? Cereb Cortex 27(6):3471-3484

Roberts JA, Perry A, Lord AR, Roberts G, Mitchell PB, Smith RE, Breakspear M (2016) The contribution of geometry to the human connectome. NeuroImage 124:379-393

Rosa MGP, Tweedale R (2005) Brain maps, great and small: lessons from comparative studies of primate visual cortical organization. Philos Trans R Soc Lond Ser B Biol Sci 360(1456):665-691

Rubinov M, Ypma RJF, Watson C, Bullmore ET (2015) Wiring cost and topological participation of the mouse brain connectome. Proc Natl Acad Sci USA 112(32):10032-10037

Sanides F (1969) Comparative architectonics of the neocortex of mammals and their evolutionary interpretation. Ann N Y Acad Sci 167(1):404-423

Schönwiesner M, Dechent P, Voit D, Petkov CI, Krumbholz K (2015) Parcellation of human and monkey core auditory cortex with fMRI pattern classification and objective detection of tonotopic gradient reversals. Cereb Cortex 25(10):3278-3289

Shipp S (2003) The functional logic of cortico-pulvinar connections. Philos Trans R Soc Lond Ser B Biol Sci 358(1438):1605-1624

Slater H, Milne AE, Wilson B, Muers RS, Balezeau F, Hunter D, Petkov CI (2016) Individually customisable non-invasive head immobilisation system for non-human primates with an option for voluntary engagement. J Neurosci Methods 269:46-60

Song HF, Kennedy H, Wang X-J (2014) Spatial embedding of structural similarity in the cerebral cortex. Proc Natl Acad Sci USA 111(46):16580-16585

Sowell ER, Thompson PM, Leonard CM, Welcome SE, Kan E, Toga AW (2004) Longitudinal mapping of cortical thickness and brain growth in normal children. J Neurosci 24(38):8223-8231

Toga AW, Thompson PM, Sowell ER (2006) Mapping brain maturation. Trends Neurosci 29(3):148-159 
van Hartevelt TJ, Kringelbach ML (2012) Chapter 34-the olfactory system. In: Mai JK, Paxinos G (eds) The human nervous system (Third Edition). Academic Press, San Diego, pp 1219-1238

Vértes PE, Alexander-Bloch AF, Gogtay N, Giedd JN, Rapoport JL, Bullmore ET (2012) Simple models of human brain functional networks. Proc Natl Acad Sci USA 109(15):5868-5873

Wagstyl K, Ronan L, Goodyer IM, Fletcher PC (2015) Cortical thickness gradients in structural hierarchies. NeuroImage 111:241-250

Wilson B, Kikuchi Y, Sun L, Hunter D, Dick F, Smith K, Petkov CI (2015) Auditory sequence processing reveals evolutionarily conserved regions of frontal cortex in macaques and humans. Nat Commun 6:8901

Xu T, Yang Z, Jiang L, Xing X-X, Zuo X-N (2015) A connectome computation system for discovery science of brain. Sci Bull 60(1):86-95

Xu T, Falchier A, Sullivan E, Linn G, Ramirez J, Ross D, Milham M (2017) Delineating the macroscale areal organization of the macaque cortex in vivo. bioRxiv. https://doi.org/10.1101/155952 